\title{
The salivary gland as a target for enhancing immunization response
}

Todd A. Ponzio ${ }^{1,2}$ and John W. Sanders $3,4^{*}$

\begin{abstract}
Background: An organism's immune response to a vaccine is dependent on a number of factors, including the site of immunization. While muscle is the most common site for vaccine administration, other sites, including the salivary gland, are poised to confer stronger and broader immunoprotection.

Findings: Studies exploring the salivary gland as an immunization site have involved protein antigens, as well as live pathogens and DNA vaccines. While intraductal instillation of protein antigens into the salivary gland may result in a relatively transient increase in antibody production, DNA or attenuated pathogen vaccination appear to confer a lasting widespread mucosal immune response that includes robust salivary and enteric IgA, as well as high levels of circulating lgG. Furthermore, vaginal and lung antibodies are also seen. For enteric pathogens, a common class of pathogen encountered by travelers, this type of immune response provides for a level of redundant protection against foreign microbes with mucosal targets.

Conclusion: The strength of immune response conferred by salivary gland vaccination is generally stronger than that seen in response to the same vaccine at a comparison site. For example, where other routes fail, immunization of the salivary gland has been shown to confer protection in lethal challenge models of infectious pathogens. A host of vaccines currently under development suffer from immunogenicity challenges, adding to the widespread interest and search for novel routes and adjuvants. With its capability to facilitate a strong and broad immune response, the salivary gland warrants consideration as an immunization site, especially for vaccines with immunogenicity challenges, as well as vaccines that would benefit from combined systemic and mucosal immunity.
\end{abstract}

Keywords: Vaccine, Immunization, Intramuscular, Salivary gland, Mucosal immunity, Enteric vaccine

\section{Background}

Vaccine delivery leading to systemic antibody-mediated immunity is usually administered through intramuscular injection (IM). Although the resulting humoral immune response forms the basis for protection conferred by nearly all vaccines delivered today, immune responses to the vaccines for bacille Calmette-Guerin (tuberculosis) and zoster trigger a strong cell-mediated immunity component [1]. Most pathogens, however, enter the body through mucosal tissues, including the aerodigestive and urogenital tracts, the eye conjunctiva and externalinterfacing exocrine glands. Given the constant interaction

\footnotetext{
* Correspondence: jwsander@wakehealth.edu

${ }^{3}$ Wake Forest University School of Medicine, Medical Center Blvd. Winston-Salem, NC 27157, USA

${ }^{4}$ Hefner Veterans Affairs Medical Center, Salisbury, NC, UK

Full list of author information is available at the end of the article
}

with the environment, the mucosal immune system has evolved efficient mechanisms to maintain tolerance toward self-antigens as non-pathogenic environmental antigens, as well as mount active immune responses against salient pathogens-features which make mucosal interfaces particularly attractive sites for vaccine delivery [2].

While most vaccines continue to be administered parenterally, a number of mucosal vaccines are commercially available. The two routes of administration for the currently licensed mucosal vaccines are oral and nasal, and examples of orally-administered vaccines include vaccines for rotavirus, poliovirus, and cholera [3]. Mucosal vaccines for various influenza viruses are delivered intranasally, and a recent exploratory clinical trial comparing a live attenuated influenza vaccine (FluMist ${ }^{\circ}$ ) administered via the intranasal or sublingual route reported haemagglutination inhibition titers of $440(n=20)$ following intranasal delivery, and 380 
( $n=20)$ following sublingual delivery [4]. The administration site also affects the amount of compartmentalization of the resulting mucosal immune response, with the sublingual route leading to broader immune responses as compared to oral and nasal administration [5]. Anatomically related to the sublingual route, another lesser-explored mucosal vaccination route includes intraductal instillation of the salivary gland.

There are three major bilateral salivary glands, the parotid, submandibular, and sublingual (collectively referred to as the SG). They are encapsulated exocrine glands responsible for the manufacturing of saliva, a complex fluid that includes significant amounts of peptides, proteins, and carbohydrates. The SG is also an immune organ, constantly coming in contact with external pathogens, and may be an optimal immunization site for certain vaccines. In addition to containing dendritic cells, a type of antigen presenting cell, the SG also appears to be a site of $B$ cell activation and isotype switching, along with induction of antigen-specific cytotoxic T lymphocytes $[6,7]$.

\section{Whole-cell killed pathogen immunization of the salivary gland}

Antibodies have long known to be present in saliva, though they have largely been viewed as potential byproduct surrogate markers of exposure to various infectious diseases [8-10]. Varying consistency across readings and individuals has made the use of saliva as a diagnostic a challenge. However, IgA from targeted SG secretions, rather than whole saliva, has been shown to serve as a high-sensitivity readout for enteric infections, including enterotoxigenic Escherichia coli [11]. This will likely become a useful proxy in field trials for vaccines targeting enteric pathogens given that collecting and assaying targeted saliva is less resource-intensive than intestinal lavages or fecal samples.

While immunizations at various sites can result in measurable changes in salivary antibodies, both serum and salivary antibodies have been reported to result from repeated intraductal instillation of either protein or formalin-fixed pathogen into the SG. One early study found a strong IgA response following the injection of bovine serum albumin (BSA) into surgically-exposed SG [12]. However, the surgical procedure itself induces a natural immune and inflammatory response, and injections via needles traverse the connective tissue sheathing of the gland. A more direct and gentle route of applying a protein antigen was subsequently explored by an Israeli group.

With an aim toward gaining a better understanding of inflammatory processes in the parotid gland, the group assessed locally-secreted antibodies in response to antigen instillation of the parotid gland. Specifically, the group investigated both salivary and systemic antibodies resulting from intraductal instillation of BSA [13, 14]. The BSA was administered repeatedly a total of eight times, and serum and saliva samples were assayed for hemagglutinating anti-BSA antibodies. While they did not evaluate the relative changes in IgAs and IgGs, an increase in overall antibody titers of the saliva was seen. However, they reported only approximately one third of the animals had systemic hemagglutinating anti-BSA antibodies, a result that may relate to the use of BSA as the immunogen, which is a component in rodent food pellets, and therefore a potential immune toleragen $[15,16]$. The ability for the SG to mount an immune response to protein or protein subunit antigens continues to remain an open question.

Around the same time as the Israeli group was exploring correlates of antigen-induced parotiditis, there was broad scientific effort in trying to develop a vaccine for dental caries, and specifically against the main offender, Streptococcus mutans. Formalin-killed S. mutans was being injected by various routes by different groups in an attempt to vaccinate various animal models from dental caries, including intravenously and subcutaneously in the mouth [17]. One group thought to investigate repeat intraductal instillation of non-human primate parotid gland. Following multiple oral subcutaneous immunization, the group then instilled formalized $S$. mutans bilaterally into the parotid glands of non-human primates [18]. While the subcutaneous immunization (total of 10 repeat administrations) led to modest increases in circulating IgM and IgG, the same subcutaneous immunizations did not change salivary IgA. However, intraductal instillation significantly increased both systemic IgG as well as salivary IgA. Buoyed by the results, the same group followed up with a study to assess efficacy (as measured by colonization), choosing to focus on immunizing only via intraductal instillation [19]. Both the number of infected surfaces, as well as the number of $S$. mutans organisms in a particular plaque were reduced in the immunized group.

A study comparing multiple subcutaneous immunizations in the vicinity of the salivary glands with multiple bilateral immunizations via intraductal instillation found the subcutaneous route induced only a serum response while intraductal instillation resulted in both serum and salivary IgA responses [20]. This finding was then later corroborated in a study instilling formalized S. mutans three times in a single parotid [21]. While these results were indeed promising, elevated specific antibody titers did not last long; and a commercial vaccine that required routine boosting would be a challenge from a commercial perspective. Live vaccines can confer more enduring protection, but due to the safety concerns related to live vaccines, DNA vaccines present themselves as promising alternatives, provided that any given DNA vaccine is sufficiently immunogenic [22]. Immunogenicity, 
however, is one of the more common challenges associated with DNA vaccines, and in this regard, the SG may have tempting appeal.

\section{Genetic vaccination of the salivary gland}

The first study to target the SG as a potential immunization site using plasmid DNA (pDNA) employed an expression system coding for Porphyromonas gingivalis fimbriae [23]. Strong humoral and cellmediated immune responses were seen in BALB/c mice following transcutaneous targeted salivary gland (TTSG) immunization (see Table 1). Though similar systemic titers of IgG were seen in response to IM or TTSG immunization, only TTSG elicited strong levels of salivary IgA. A more direct route of delivering a genetic vaccine to the SG was subsequently explored by a different group using a plasmid coding for the influenza NP protein. In this pilot study, pDNA complexed with the cationic lipid reagent adjuvants GAP-DLRIE/DOPE or Vaxfectin $^{\circ}$ was instilled intraductally into the SG in rats
[24]. While the authors were unable to detect changes in salivary IgA, significant changes in systemic IgG were observed.

A more extensive study, also done in rats, involved the bilateral instillation of $88 \mu \mathrm{g}$ of pDNA coding for either of three antigens, human growth hormone (hGH), HIV envelope protein gp120, or protective antigen (PA) from Bacillus anthracis (i.e., anthrax) [25]. As compared to IM and sublingual vaccination, higher titers of systemic IgG (and IgA) were seen in response to $\mathrm{SG}$ vaccination of plasmid encoding for hGH. Higher titers in response to SG, rather than IM, vaccination were observed for the gp120 genetic vaccine as well, and a memory response was observed. Circulating antibodies titers in response to SG and subcutaneous vaccination were more than ten-fold higher than those seen in response to IM vaccination with the PA gene; and the higher titers (but not the lower titers seen following IM vaccination) provided protection from a subsequent lethal anthrax challenge. Of particular interest to those involved in the

Table 1 A summary of various experiments using the Salivary Gland as an immunization site

\begin{tabular}{|c|c|c|c|c|c|}
\hline Antigen & IgA, saliva & IgG, systemic & Reference & Model & Notes \\
\hline $\begin{array}{l}\text { Formalized } \\
\text { S. mutans }\end{array}$ & ++++ & ++++ & [18] & $\mathrm{NHP}$ & $\begin{array}{l}\text { SGid > SC; Greater IgA and IgG seen with SGid imzn } \\
\text { than with SC imzn. }\end{array}$ \\
\hline $\begin{array}{l}\text { Formalized } \\
\text { S. mutans }\end{array}$ & +++ & +++ & [21] & $\mathrm{NHP}$ & Greater IgA seen with SGid imzn than with SC imzn. \\
\hline $\begin{array}{l}\text { Formalized } \\
\text { S. mutans }\end{array}$ & ++++ & ++++ & [19] & $\mathrm{NHP}$ & $\begin{array}{l}\text { SGid imzn resulted in semi-protection against a } S \text {. mutans } \\
\text { challenge. }\end{array}$ \\
\hline $\begin{array}{l}\text { Formalized } \\
\text { S. mutans }\end{array}$ & ++++ & +++ & [20] & $\mathrm{NHP}$ & SGid immunization was strain specific. \\
\hline Bovine serum albumin & $++^{a}$ & $++^{a}$ & [13] & Rats & $\begin{array}{l}\text { SGid; Moderate salivary and systemic antibody responses } \\
\text { reported }\end{array}$ \\
\hline Bovine serum albumin & $++^{a}$ & $t^{a}$ & [14] & Rats & $\begin{array}{l}\text { Multiple SGid BSA imzns are followed by systemic } \\
\text { antibodies. }\end{array}$ \\
\hline MCMV, tCMCMV & n.a. & ++++ & [33] & Mice & $\begin{array}{l}\text { SGig imzn resulted in protection from viremia; IP } \\
\text { imzn did not. }\end{array}$ \\
\hline MCMV, tcMCMV & +++ & +++ & [7] & Mice & $\begin{array}{l}\text { SGig > IP; Greater IgA and IgG seen with SGig imzn than } \\
\text { with IP imzn. }\end{array}$ \\
\hline Adenovirus & +++ & ++++ & [15] & Rats & $\begin{array}{l}\text { SGid; Repeated pre-exposure to inactivated adenovirus } \\
\text { induced immune tolerance. }\end{array}$ \\
\hline tcMCMV & +++ & ++++ & [34] & Mice & SGid imzn resulted in protection from a lethal challenge. \\
\hline $\begin{array}{l}\text { pDNA: fimbriae from } \\
\text { P. ginigivalis }\end{array}$ & ++++ & +++ & [23] & Mice & $\begin{array}{l}\text { TSG > IM; Greater IgA seen with TSG imzn, than with IM } \\
\text { imzn. TSG and IM imzns resulted in equal IgG. }\end{array}$ \\
\hline pDNA: influenza NP protein & n.c. & +++ & [24] & Rats & SGid; Included testing of two adjuvants. \\
\hline $\begin{array}{l}\text { pDNA: NP protein, hGH, } \\
\text { gp120, anthrax PA }\end{array}$ & ++++ & ++++ & [25] & Rats & $\begin{array}{l}\text { SGid }>\text { IM; Greater IgA and IgG seen with SGid imzn than with } \\
\text { IM imzn. SG imzn protected from a lethal challenge; IM imzn } \\
\text { did not; distal mucosal response seen. }\end{array}$ \\
\hline pDNA: gp120 & +++ & +++ & [27] & Rats, dogs & $\begin{array}{l}\text { SGid }>\text { IM (ASCs from Peyer's patches); distal mucosal IgA } \\
\text { response seen in lungs. }\end{array}$ \\
\hline
\end{tabular}

Abbreviations: SGid intraductal instillation of the salivary gland, SGig direct injection into the gland through a small incision, TSG targeted injection of the salivary gland, SC subcutaneous injection, IM intramuscular injection, IP intraperitoneal injection, imzn immunization, ASC antibody secreting cell, NHP non-human primate, MCMV murine cytomegalovirus, tCMCMV tissue-cultured MCMV (i.e., attenuated MCMV), anthrax PA anthrax protective antigen, $h G H$ human growth hormone, gp120 HIV gp120, pDNA plasmid DNA, n.c. no change, n.a. not addressed. ${ }^{a}$ Antibody response confounded by immunogen being present in animal chow, possibly inducing immune tolerance; see $[15,16]$. Table compiled by searching the PubMed database on "instillation duct antibodies" or "intraductal instillation antibodies," analyzing salient peer-reviewed reports and their references, then subjecting those reports to a citation tracking database (Web of Science ${ }^{\oplus}$ ) and analyzing the resulting reports, which were also run through a citation tracking database, with the salient reports evaluated as well 
development of novel mucosal vaccines were the observations of widespread mucosal antibody responses and significant increases in salivary and fecal IgA, as well as lung and vaginal IgG following SG vaccination with gp120 combined with a Zn/lipid adjuvant. Antibodysecreting cells (ASCs) isolated from Peyer's patches following SG vaccination were approximately four times higher than those seen following IM vaccination. As antibody titer can predict efficacy, even for cell-mediated immunity, these results suggest it would be prudent to include the SG as a possible vaccination site [26].

Lastly, in an effort to explore the scalability of intraductal instillation of a DNA vaccine to larger animals, the method was extended to beagles in a follow-up study [27]. Solutions containing pDNA in the presence of $\mathrm{Zn}$ (with or without lipid) were instilled into the SG of the rats and beagles. The rats and dogs received bilateral instillations of $88 \mu \mathrm{g}$ and $2.5 \mathrm{mg}$ of pDNA, respectively, and the resulting antibody titers following SG vaccination were similarly high, registering at above 10,000. Addition of $\mathrm{Zn}$ improved protein expression, and a distal IgA response was also measured in the lungs. ASCs from Peyer's patches were evaluated in this study as well, and SG immunization was seen to result in approximately 3-4 times as many ASCs as compared to IM immunization. Furthermore, there have been many described improvements related to the vector design of DNA vaccines, and optimizing future plasmids for instillation would only further enhance effectiveness [28]. Collectively, the data suggest the SG may warrant inclusion as a test vaccination route for mucosal vaccines under development, especially DNA vaccines that would benefit from increased immunogenicity.

\section{Live pathogen vaccination of the salivary gland}

Though the SG is a well-established effector site for antibody-secreting B cells, induction of B cells is generally more restricted to specific sites, such as mucosaassociated lymphoid tissue of the ileum [29]. However, infiltrating leukocytes in other tissues (including SG) can form ectopic lymphoid-like structures, including organized aggregates that promote antigen-specific adaptive immune responses to include the proliferation of B cells and the production of antigen-specific antibodies [30]. In response to an immune challenge, the SG can form ectopic lymphoid-like structures that serve as inductive sites and which express activation-induced cytidine deaminase (AID), an enzyme required for somatic hypermutation and class switching [7]. This understanding helps explain what happens when mechanisms get misaligned, as in Sjogren's syndrome, as well as the opportune results from studies instilling live pathogens [31].

Vaccination using live pathogens has long been demonstrated to elicit broad and lasting immune responses.
Indeed, the first vaccines administered were all live [32]. In an effort to explore distal immune responses following SG inoculation, one study investigated tissue viral titers, tissue pathology, and antibody responses. The inoculum consisted of either murine cytomegalovirus (MCMV) or the less virulent tissue-culture-derived MCMV (tcMCMV) delivered through various routes, including injecting into the salivary gland through a small incision (intraglandular) [33]. While systemic IgG and IgM observed in response to intraglandular and intraperitoneal inoculation were similar, of all the routes tested (intraperitoneal, intranasal, periglandular and intraglandular), only intraglandular SG inoculation of tcMCMV was able to prevent splenic necrosis and hepatitis while limiting SG viral titers. Delivering tcMCMV into the SG was also shown to correlate with maintaining SG leukocyte infiltrates.

A subsequent comprehensive study by the same group employed the gentler route of intraductal instillation. Here, instilling either a replication-deficient recombinant adenovirus expressing individual MCMV genes or tcMCMV resulted in protection against a lethal systemic challenge with MCMV [34]. Due to its broad tropism and relative safety, the adenovirus is a common vector used to deliver vaccine antigens and other transgenes, and its potent immune reactions in the SG can be tolarized through pre-exposure [35]. Using a recombinant replication-deficient adenovirus to deliver MSMV genes resulted in strong systemic neutralizing MCMV-specific IgG in the serum [34]. Additionally, immunization with the adenovirus induced mucosal neutralizing MCMVspecific IgA at the primary immunization site (saliva), as well as distal mucosal sites (feces and vagina). This observation further corroborated and extended the findings by Tucker et al 2003, that SG vaccination with pDNA results in distal mucosal immune responses. Lastly, these immune responses provided vital protection in a lethal MCMV challenge. In light of the strong mucosal and systemic protective responses, the findings support the further testing of the salivary gland as a potentially attractive platform for active vaccine delivery.

\section{Conclusion}

Here we have attempted to provide an overview of the SG as an immunization site, and synthesize a number of studies suggesting it be included as a selected immunization site in the development of novel vaccines, especially vaccines that do not elicit strong immune responses. Table 1 provides a qualitative analysis of salivary and systemic antibody responses following immunization of the salivary gland in several animal models. Antibody responses were measured by a host of methods, including hemagglutinating antibody titers, indirect immunofluorescent staining of the immunogen, ELISA optical density, and percent 
specific antibody values measured by radioimmunoassay, and a general qualitative magnitude of systemic IgG and salivary IgA response is provided.

As shown in the Table, various groups have demonstrated the SG to be capable of eliciting robust widespread mucosal and systemic protective responses in following antigen presentation, routinely resulting in antibody titers higher than those seen with other immunization targets. It is notable that studies have generally instilled the particular immunogen or vector (e.g. formalized S. mutans, BSA, virus, or plasmid DNA) in the absence of an adjuvant, yet still observed significant immune responses. Indeed, while complete Freund's adjuvant has been used in studies employing subcutaneous immunizations delivered in the vicinity of the salivary glands (reviewed in [17]), very few studies using intraductal instillation have included potential immune adjuvants in the infusate (cationic lipid transfection reagents Vaxfectin $^{\circ}$ and GAP-DLRIE/DOPE in rats, or azo dye Evans blue in dogs) [24, 27]. Studies have generally instilled the test vaccine in a saline vehicle, achieving notable immune responses in the absence of an adjuvant. Given that vaccine efficacy is typically linked to antibody titer concentration, achieving high titers would be a common goal. A host of groups are exploring novel adjuvants to enhance immunogencitiy, the needs of which may be diminished if the vaccination site were able to facilitate a strong enough immune response.

While intraductal instillation into the SG is not an obvious route for vaccination, the potential benefits seen by a robust immune response would warrant the additional research effort. The practicality of mass immunization via the SG may be logistically challenging. However, infusion of the gland is a relatively simple procedure that can be done in an outpatient setting, such as during a routine dental check-up. While intraductal instillation is quick and painless, requiring neither needles nor anesthesia, most health-care workers have only modest familiarity with the procedure; and so training and instrumentation advances that simplify vaccine administration would significantly increase the amenability of the SG as a preferred site.

Additionally, saliva contains a number of carbohydrates, peptides, and enzymes, and this complex mixture may require novel formulation approaches to maximize vaccine efficiency and bring an SG-based vaccine into practical application. While the infusate vehicle in most SG immunization studies has been either water or a simple saline solution, the inclusion of mucoadhesives, protease, or nuclease inhibitors could further increase immunization efficiency. For example, adding zinc to the ductal infusate has been reported to result in a 20 -fold increase in transgene expression, which would be important for DNA-based vaccines [36]. Nevertheless, the studies described above demonstrate striking capabilities of the SG as an immunization site. There are currently a host of antigen-based mucosal vaccines under development using live-attenuated pathogens, as well as DNAbased, carbohydrate, and protein antigens. While further studies are certainly needed to build upon the current literature discussed here, and specifically to compare SG intraductal instillation with other administration techniques into other tissues, the SG presents as an attractive route of administration.

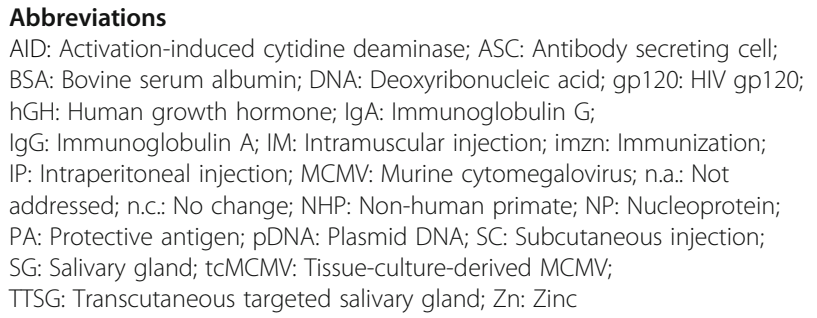

Acknowledgements

Not applicable.

Funding

No external funding was received for the preparation of this review.

Availability of data and materials

Not applicable.

Authors' contributions

TP and JS contributed equally to the development of this article. Both authors read and approved the final manuscript.

\section{Competing interests}

The authors have no competing interests to declare.

Consent for publication

TP and JS have both given final approval of the version to be published and agreed to be accountable for all aspects of the work.

Ethics approval and consent to participate

Not applicable.

\section{Declarations}

The views expressed in this article are of the authors and do not necessarily reflect official policy or position of the DoD, VA, or US Government. No copyright protection is available for this work under 17 USC 105.

\section{Author details}

${ }^{1}$ Naval Medical Research Center, 503 Robert Grant Ave., Silver Spring, MD 20910, USA. Wake Forest University School of Medicine, Winston-Salem, USA. ${ }^{3}$ Wake Forest University School of Medicine, Medical Center Blvd., Winston-Salem, NC 27157, USA. ${ }^{4}$ Hefner Veterans Affairs Medical Center, Salisbury, NC, UK.

Received: 9 February 2017 Accepted: 13 February 2017

Published online: 17 February 2017

\section{References}

1. Plotkin SA. Correlates of protection induced by vaccination. Clin vaccine immunol. 2010;17(7):1055-65.

2. Holmgren J, Czerkinsky C. Mucosal immunity and vaccines. Nat Med. 2005; 11 (4 Suppl):S45-53.

3. Lycke N. Recent progress in mucosal vaccine development: potential and limitations. Nat Rev Immunol. 2012;12(8):592-605. 
4. Yu K-S, Czerkinsky C. Immunogenicity and Safety of Live Attenuated Influenza Vaccine (Flumist) Administered by Nasal and Sublingual Route. In: ClinicalTrials gov. 5Nov2013 edn: U.S. National Institutes of Health; 2013.

5. Czerkinsky C, Cuburu N, Kweon MN, Anjuere F, Holmgren J. Sublingual vaccination. Hum Vaccin. 2011;7(1):110-4.

6. Stasulis CA, Hand AR. Immunohistochemical identification of antigen presenting cells in rat salivary glands. Arch Oral Biol. 2003;48(10):691-9.

7. Grewal JS, Pilgrim MJ, Grewal S, Kasman L, Werner P, Bruorton ME, London SD, London L. Salivary glands act as mucosal inductive sites via the formation of ectopic germinal centers after site-restricted MCMV infection. Faseb J. 2011;25(5):1680-96.

8. Ellison SA, Mashimo PA, Mandel ID. Immunochemical studies of human saliva. I. The demonstration of serum proteins in whole and parotid saliva. J Dent Res. 1960;39:892-8.

9. Hofman LF. Human saliva as a diagnostic specimen. J Nutr. 2001;131(5): 1621S-5S

10. Exum NG, Pisanic N, Granger DA, Schwab KJ, Detrick B, Kosek M, Egorov Al, Griffin SM, Heaney CD. Use of pathogen-specific antibody biomarkers to estimate waterborne infections in population-based settings. Curr Environ Health Rep. 2016;3(3):322-34.

11. Aase A, Sommerfelt $H$, Petersen LB, Bolstad M, Cox RJ, Langeland $N$, Guttormsen AB, Steinsland H, Skrede S, Brandtzaeg P. Salivary IgA from the sublingual compartment as a novel noninvasive proxy for intestinal immune induction. Mucosal Immunol. 2016;9(4):884-93.

12. Emmings FG, Genco RJ. The IgA antibody-forming cell response in the rabbit submandibular gland following several different methods of immunization. Adv Exp Med Biol. 1974;45:467-71.

13. Boss JH, Rosenmann, Sela J, Ulmansky, Dishon T. Antibodies in the saliva and serum of rats sensitized by intraductal instillation of antigen into the parotid gland. J Dent Res. 1975;54(3):609-14.

14. Dishon T, Sela J, Ulmansky M, Rosenmann E, Boss JH. Antibodies in the saliva after antigenic challenge of the parotid gland of systemically sensitized rats. Med Microbiol Immunol. 1975;161(1):47-52.

15. Kagami H, Atkinson JC, Michalek SM, Handelman B, Yu S, Baum BJ, O'Connell B. Repetitive adenovirus administration to the parotid gland: role of immunological barriers and induction of oral tolerance. Hum Gene Ther. 1998;9(3):305-13.

16. Dishon T, Sela J, Ulmansky M, Rosenmann E, Boss JH. Naturally occurring antibodies to bovine serum albumin in the parotid saliva of rats. Arch Oral Biol. 1975;20(7):415-8.

17. Smith DJ, Taubman MA. Immunization experiments using the rodent caries model. Journal of dental research. 1976. 55 Spec No:C193-205.

18. Emmings FG, Evans RT, Genco RJ. Antibody response in the parotid fluid and serum of Irus monkeys (Macaca fascicularis) after local immunization with Streptococcus mutans. Infect Immun. 1975;12(2):281-92.

19. Evans RT, Emmings FG, Genco RJ. Prevention of Streptococcus mutans infection of tooth surfaces by salivary antibody in Irus monkeys (Macaca fascicularis). Infect Immun. 1975;12(2):293-302.

20. Emmings FG, Evans RT, Genco RJ: Immunization of Macaca fascicularis (Macaca irus) monkeys with Streptococcus mutans: specificity of antibody responses in saliva. Journal of dental research. 1976. 55 Spec No:C181-192.

21. Walker J. Antibody responses of monkeys to oral and local immunization with Streptococcus mutans. Infect Immun. 1981;31(1):61-70.

22. Ferraro B, Morrow MP, Hutnick NA, Shin TH, Lucke CE, Weiner DB. Clinical applications of DNA vaccines: current progress. Clin Infect Dis. 2011;53(3): 296-302.

23. Kawabata S, Terao Y, Fujiwara T, Nakagawa I, Hamada S. Targeted salivary gland immunization with plasmid DNA elicits specific salivary immunoglobulin $A$ and $G$ antibodies and serum immunoglobulin $G$ antibodies in mice. Infect Immun. 1999;67(11):5863-8.

24. Sankar V, Baccaglini L, Sawdey M, Wheeler CJ, Pillemer SR, Baum BJ, Atkinson JC. Salivary gland delivery of pDNA-cationic lipoplexes elicits systemic immune responses. Oral Dis. 2002;8(6):275-81.

25. Tucker SN, Lin K, Stevens S, Scollay R, Bennett MJ, Olson DC. Systemic and mucosal antibody responses following retroductal gene transfer to the salivary gland. Mol ther. 2003;8(3):392-9.

26. Spellberg B, Ibrahim AS, Lin L, Avanesian V, Fu Y, Lipke P, Otoo H, Ho T, Edwards Jr JE. Antibody titer threshold predicts anti-candidal vaccine efficacy even though the mechanism of protection is induction of cellmediated immunity. J Infect Dis. 2008;197(7):967-71.

27. Tucker SN, Lin K, Stevens S, Scollay R, Bennett MJ, Olson DC. Salivary gland genetic vaccination: a scalable technology for promoting distal mucosal immunity and heightened systemic immune responses. Vaccine. 2004; 22(19):2500-4.

28. Williams JA. Improving DNA vaccine performance through vector design. Curr Gene Ther. 2014;14(3):170-89.

29. Thrane PS, Sollid LM, Haanes HR, Brandtzaeg P. Clustering of IgA-producing immunocytes related to HLA-DR-positive ducts in normal and inflamed salivary glands. Scand J Immunol. 1992;35(1):43-51.

30. Pitzalis C, Jones GW, Bombardieri M, Jones SA. Ectopic lymphoid-like structures in infection, cancer and autoimmunity. Nat Rev Immunol. 2014; 14(7):447-62.

31. Astorri E, Scrivo R, Bombardieri M, Picarelli G, Pecorella I, Porzia A, Valesini G, Priori R. CX3CL1 and CX3CR1 expression in tertiary lymphoid structures in salivary gland infiltrates: fractalkine contribution to lymphoid neogenesis in Sjogren's syndrome. Rheumatology (Oxford). 2014;53(4):611-20.

32. Plotkin SA. Vaccination against the major infectious diseases. C R Acad Sci III. 1999;322(11):943-51.

33. Pilgrim MJ, Kasman L, Grewal J, Bruorton ME, Werner P, London L, London SD. A focused salivary gland infection with attenuated MCMV: an animal model with prevention of pathology associated with systemic MCMV infection. Exp Mol Pathol. 2007;82(3):269-79.

34. Liu G, Zhang F, Wang R, London L, London SD. Protective MCMV immunity by vaccination of the salivary gland via Wharton's duct: replication-deficient recombinant adenovirus expressing individual MCMV genes elicits protection similar to that of MCMV. Faseb J. 2014:28(4):1698-710.

35. Hoque AT, Liu X, Kagami H, Swaim WD, Wellner RB, O'Connell BC, Ambudkar IS, Baum BJ. Construction and function of a recombinant adenovirus encoding a human aquaporin 1-green fluorescent protein fusion product. Cancer Gene Ther. 2000;7(3):476-85.

36. Niedzinski EJ, Olson DC, Chen YJ, Udove JA, Nantz MH, Tseng HC, Bolaffi JL, Bennett MJ. Zinc enhancement of nonviral salivary gland transfection. Mol ther. 2003;7(3):396-400.

\section{Submit your next manuscript to BioMed Central and we will help you at every step:}

- We accept pre-submission inquiries

- Our selector tool helps you to find the most relevant journal

- We provide round the clock customer support

- Convenient online submission

- Thorough peer review

- Inclusion in PubMed and all major indexing services

- Maximum visibility for your research

Submit your manuscript at www.biomedcentral.com/submit
) Biomed Central 